\title{
Orthotic design through 3D reconstruction: A passive-assistance ankle-foot orthotic
}

doi:10.1533/abbi.2005.0014

\author{
A. L. Darling and W. Sun \\ Department of Mechanical Engineering and Mechanics, Drexel University, Philadelphia, Pennsylvania, USA
}

\begin{abstract}
Current methods of designing and manufacturing custom orthotics include manual techniques such as casting a limb in plaster, making a plaster duplicate of the limb to be treated and forming a polymer orthotic directly onto the plaster model. Such techniques are usually accompanied with numerous postmanufacture alterations to adapt the orthotic for patient comfort. External modeling techniques rely heavily on the skill of the clinician, as the axes of rotation of any joint are partially specified by the skeletal structure and are not completely inferable from the skin, especially in cases where edema is present. Clinicians could benefit from a simultaneous view of external and skeletal patient-specific geometry. In addition to providing more information to clinicians, quantification of patient-specific data would allow rapid production of advanced orthotics, requiring machining rather than casting. This paper presents a supplemental method of orthotic design and fitting, through 3D reconstruction of medical imaging data to parameterise an orthotic design based on a major axis of rotation, shape of rigid components and placement of skin contact surfaces. An example of this design approach is shown in the design of an ankle-foot orthotic designed around the computed tomography data from the Visible Human Project.
\end{abstract}

Key words: 3D reconstruction, orthotic, ankle.

\section{INTRODUCTION}

A wide variety of conditions exist for which external reinforcement or muscular assistance would be helpful to patients in moving a limp or weakened limb. Fitting an external device to match the range of motion of a given joint can be challenging. There is a great deal of expertise required, as orthotists frequently perform such refits as blowing regions of a polymer orthotic out using a heat gun, adjusting the amount of support provided by cutting off material and selectively adding padding to adjust fit and comfort. Fitting of a custom orthotic with hinged components requiring machining can take several weeks to months (Wu et al. 2002).

In this paper, we demonstrate a method of $3 \mathrm{D}$ reconstruction to parameterise a joint structure, converting medical imaging data to an engineering model format

Corresponding Author:

W. Sun

Department of Mechanical Engineering and Mechanics

Drexel University, Philadelphia

Pennsylvania 19104, USA

Tel: +215-895-5810

Email: sunwei@drexel.edu for construction of a machined or freeform fabricated orthotic. The specific orthotic selected was an ankle orthotic for the treatment of drop foot. Drop foot is a gait disruption characterised by inability to lift the foot during the swing phase of gait, due to lack of activity in ankle dorsiflexion and toe extensor muscles or excessive activity of the plantarflexing calf muscles. This can be caused by a number of conditions, including neurological disease, stroke and spinal cord injury. Incidence of restricted ankle dorsiflexion has been reported to be as great as $76 \%$ in patient samples with brain trauma (Moseley et al. 2003). Fifteen percent of sports injuries involve the ankle joint complex, and a proportion of any lateral ligament injuries leads to chronic instability (Corazza et al. 2003). A common treatment for weakened ankle dorsiflexion is immobilisation, either through an external jointless (without a hinge) orthotic (Rubin and Cohen 1988) or through fusion surgery (Leardini 2001).

Jointed (or hinged) ankle orthotics for drop foot are less common, and they focus on immobilisation of all but one degree of freedom, that of dorsiflexion. Such an ankle-foot orthotic will assist with dorsiflexion to allow the toes to clear the ground during the swing phase of gait but will not help with plantar flexion (Fay and Boninger 2002). The joint is either constructed as a hinge or by crossing the gap between foot and calf components with a semielastic 


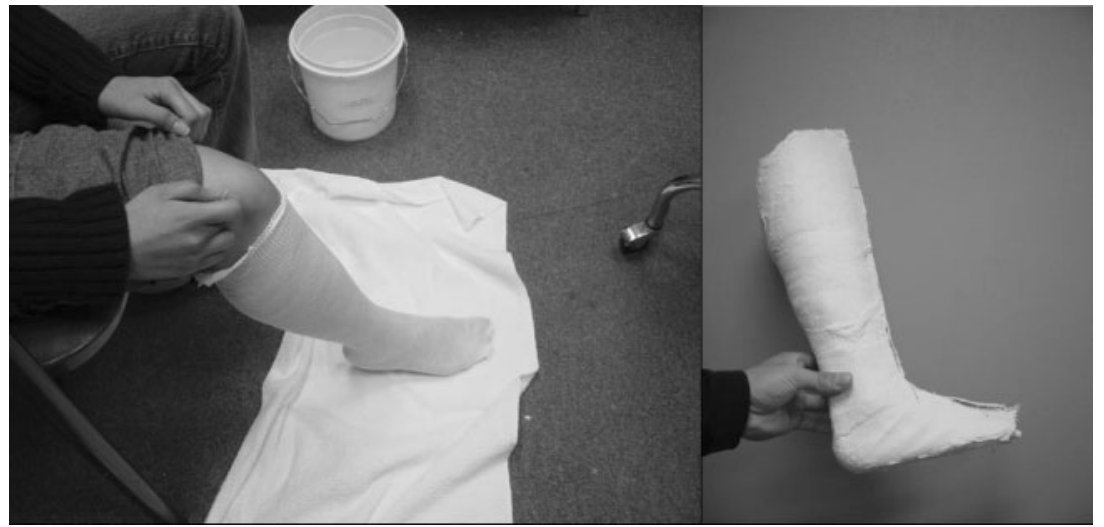

Figure 1 A conventional ankle orthotic fitting.

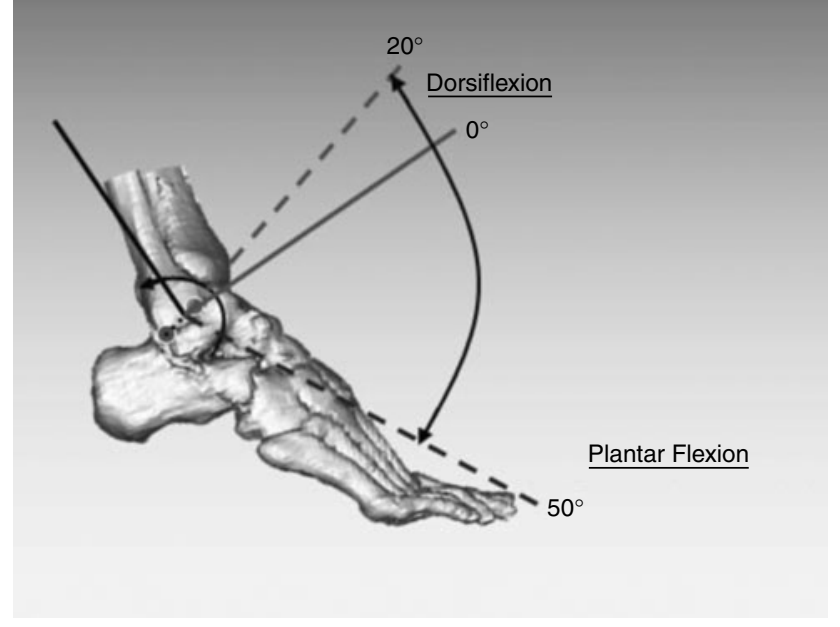

Figure 2 The ankle rotating in one degree of freedom, that of dorsiflexion/plantar flexion.

component designed to bend with the patient's weight. The fitting of the joint depends on the experience of the clinician and repetition. As shown in Figure 1, a plaster cast is first constructed of the patient's ankle, and then the cast is modified by the clinician to specify the axis of rotation and any locations that require padding. The modified cast is then used to create a solid model of the ankle, and a thermoplastic material is melted across the leg template to create a form-fitting orthotic. While this method creates aesthetic orthotics, patient visits for refitting are frequent (Goldberg and Hsu 1997).

Instead of judging the axis of rotation from the exterior view of the ankle, a $3 \mathrm{D}$ representation of the skeleton would allow the clinician to see more detail, as is the case in Figure 2. Figure 2 is a 3D reconstruction of sequential computed tomography (CT) images from the Visible Human Project (2003). The axis of rotation for dorsiflexion was estimated from the tip of the lateral malleolus to the tip of the medial malleolus (Wu et al. 2002). CT scan images are sequential axial X-ray images of a patient, and as such express density information, such as the presence of soft tissue as opposed to bone. Common clinical CT scanners are capable of submillimeter resolution in all three axes. These slices may be reconstructed into a $3 \mathrm{D}$ volumetric or CAD model, which may be subsequently measured for fitting information. The fitting information is more detailed than exterior visual inspection, as a $3 \mathrm{D}$ model would exist for both the exterior of the ankle and the skeleton within. A design model of an orthotic, once parameterised to the patient's fitting specifics, may be subsequently constructed using any of a number of freeform fabrication techniques (Sun and Lal 2002).

A single-degree-of-freedom orthotic for the ankle was selected for demonstration, not because of clinical applicability but the simplicity of a one-degree-of-freedom hinge, and for exterior landmarks of the foot, which ease orthotic design, specifically the sole of the foot and the heel. In practice, use of this technique for the ankle would be unnecessary as traditional techniques achieve success without exposing the patient to ionising radiation. Applying this technique towards three-degree-of-freedom joints, such as the hip or shoulder, would likely be more clinically applicable.

This design approach was applied to the publicly available CT scan data of the Visible Human Project (2003). Relevant joint and soft tissue parameters were measured and placed into the coordinate system of a simple hinged ankle orthotic design. Data entry of patient-specific coordinate data from the Visible Human male and Visible Human female allowed for automated resizing of the ankle orthotic design.

\section{MATERIALS AND METHODS}

\section{Design of the orthotic and coordinate system}

A design for an ankle orthotic was created with two components, one for the foot and one for the lower leg, treating each body part as a rigid component attached by a one-degree-of-freedom hinge, at the dorsiflexion axis of rotation. There are eight anchor points on the orthotic design whereby straps could be attached to hold the patient's ankle firmly in place. These anchor points were selected to allow elastic muscle assistance bands to run 


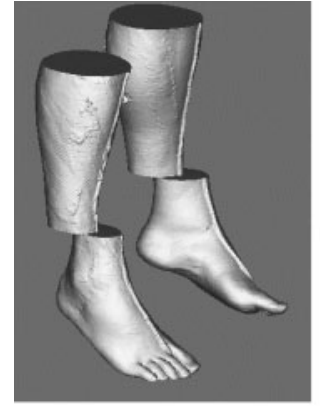

(a)

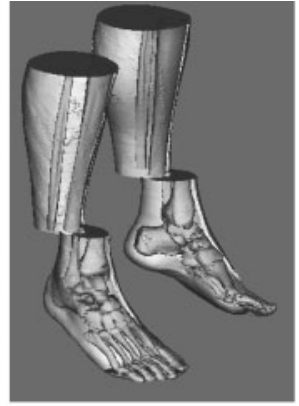

(b)

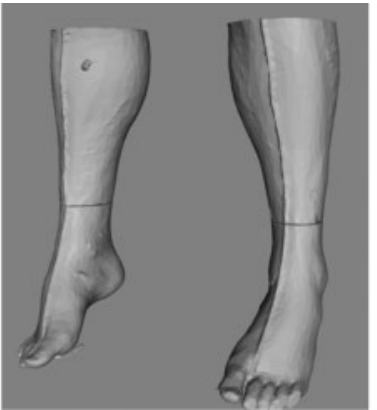

(c)

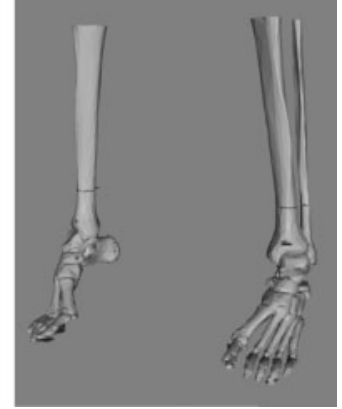

(d)

Figure 3 (a, b) 3D reconstruction of the Visible Human male CT data and (c, d) repaired 3D models, corrected for change of scanning position.

through loops on the straps and across the joint itself, creating passive dorsiflexion assistance. The design was created to illustrate the potential for standardisation for such orthotics.

In establishing a coordinate system for the model space of the orthotic, a method similar to the multiple Joint Coordinate System recommended by the Standardization and Terminology Committee of the International Society of Biomechanics was used. According to the ISB, in establishing a joint coordinate system, "First, a Cartesian coordinate system (CCS) is established for each of the two adjacent body segments. The axes in these CCSs are defined based on bony landmarks that are either palpable or identifiable from X-rays. . the common origin of both axis systems is the point of reference for the linear translation occurring in the joint, at its initial neutral position (Wu et al. 2002)." A similar orthogonal joint coordinate system was used for the orthotic, with two CSSs, but for reasons associated with exporting the model to different digital formats, the origin for these systems was moved such that the model rested entirely in the positive domain of each coordinate system.

The orthotic was designed in Pro/ENGINEER (PTC, Needham, MA). Each component was dependent on Cartesian coordinate systems, B0-S0-H0 for the foot component and C0-SH0-L0 for the lower-leg component. These base coordinate planes are intended to match anatomical features: $x y$ planes based on the sole of the foot (S0) and the axis of dorsiflexion (C0), $x z$ planes based on the posterior of the heel $(\mathrm{H} 0)$ and the anterior surface of the shin ( $\mathrm{SH} 0)$ and $y z$ planes based on the lateral edge or blade of the foot (B0) and the lateralmost edge of the lower leg (L0). All features of the orthotic were dependent on planes offset from these base coordinate (or datum) planes. The structure of the orthotic is entirely dependent on these planes with the exception of two elements, the size of the axle protrusion and the strap attachment cuts, as these elements should be standardised for other components, namely a screw component for the axles and straps for the strap attachment points. New offset distances can be entered into the Pro/ENGINEER orthotic design, and the orthotic design changes accordingly in an automated manner for each data set.

\section{Fitting data acquisition}

The goal of the fitting data acquisition was to convert the imaging data of two subjects' right legs into the coordinate system established for the orthotic model, such that the model would rescale for both individuals.

CT data for the Visible Human male and Visible Human female was downloaded from the public access Web site (Visible Human Project 2003). The format of the Visible Human data was compressed Advantage files, which included positional information as to the location of a single slice image in the stack. These files were converted into serial bitmap images using MRIcro freeware (2003). The resultant bitmap images lacked the axial positioning data of the Advantage files and enabled $3 \mathrm{D}$ reconstruction as a single entity.

$3 \mathrm{D}$ reconstruction of the sequential $512 \times 512$-pixel bitmap slices was performed using Mimics software (Materialise, Ann Arbor, MI). Two threshold masks were established in each sample set, one to isolate the soft tissue of the legs and one to isolate dense, bony tissues. Regiongrowing operations were performed to eliminate noise in the model. The 3D models are displayed in Figures 3 and 4. The distortion of the legs of the Visible Human male shown in Figure 3(c) and (d) occurred because the two regions of the leg were scanned separately using different coordinate systems. These coordinate systems were inherent in the Advantage file, necessitating the conversion of the file type described previously. The resulting 3D models for the Visible Human male needed to be repaired prior to parameterisation. The model reconstructed from the Visible Human female data required no repair.

The two 3D models per data set, one for hard tissue and one for soft tissue, were exported to stereolithography (.STL) format, and subsequently processed in Geomagic software (Geomagic Inc., Research Triangle Park, NC) to reduce the number of facets defining their surfaces. This decimation operation was performed to increase processing speed and was not judged to change the boundary representation of the soft tissue or skeleton models significantly. The models were then exported to 3D Studio mesh format (.3DS) for model visualisation, repair and parameterisation. 


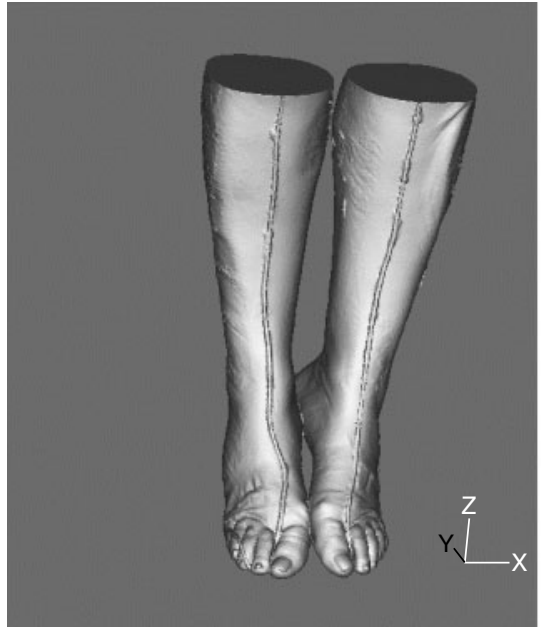

(a)

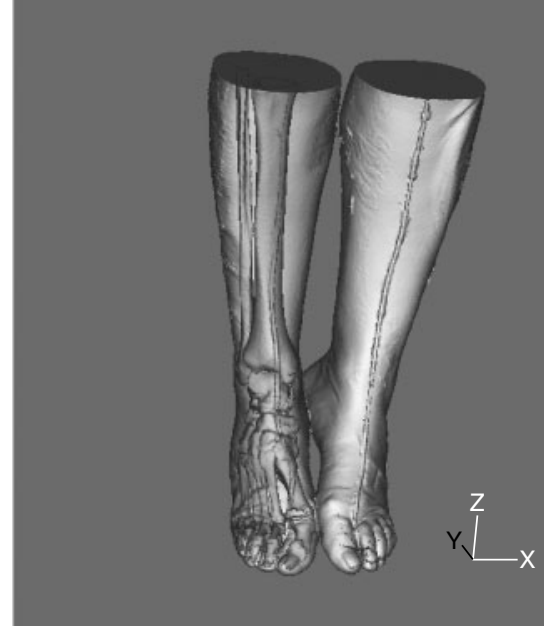

(b)

Figure 4 The 3D reconstructions of the Visible Human female: (a) the soft tissue model of both legs and (b) the soft tissue shown in conjunction with the skeleton of the right leg. No repair of the model was required.

The models, once imported into 3D Studio R 3.1 (Autodesk, San Rafael, CA), still shared a common coordinate system established in Mimics, with the soft tissue model surrounding the hard tissue model anatomically. Model repair was conducted by translating the vertices of the distorted upper leg to match landmarks on the lower leg. The specific landmarks used were the positioning wire running along the front of the leg to match $x$ and $y$ offsets and the curvature of the fibula to match the $z$ offset. The repaired models are shown in Figure 3(c) and (d). Translations to both soft tissue and skeletal models were performed simultaneously and identically to prevent any relational distortion between the models.

Parameterisation of the right foot and leg was performed for both male and female data sets, treating the foot and lower leg as separate rigid components. As the feet were positioned differently than they would be in the orthotic, two separate parameterisations were necessary, one for each foot and leg. Parameterisation was accomplished by defining planes along the model. First, an orthogonal set of planes was established, with a plane along the blade of the foot, one tangential to the heel, and one across the sole of the foot in the soft tissue model. All other fitting parameter planes were then measured in terms of offset distances from these orthogonal planes. A final set of orthogonal planes, still described in offset distances from the coordinate planes, was parameterised from the skeleton model, estimating the axis of rotation for dorsiflexion, estimated as the line between the tip of the medial malleolus and the tip of the lateral malleolus ( $\mathrm{Wu}$ et al. 2002). Images from the parameterisation of the Visible Human male model may be seen in Figure 5. A description of landmarks may be seen in Table 1 of the Results section. Table 1 also includes derived parameters, parameters created not by specific landmarks but by relations of landmark parameters. These largely relate to aesthetic aspects of the orthotic. C1 through C3

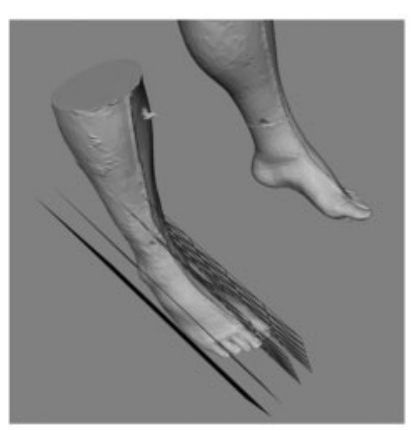

(a) the B or blade offsets

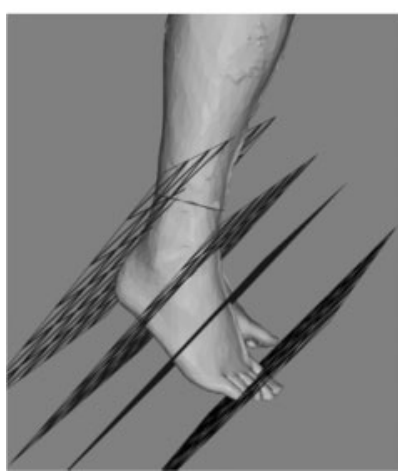

(c) the $\mathrm{H}$ or heel offsets

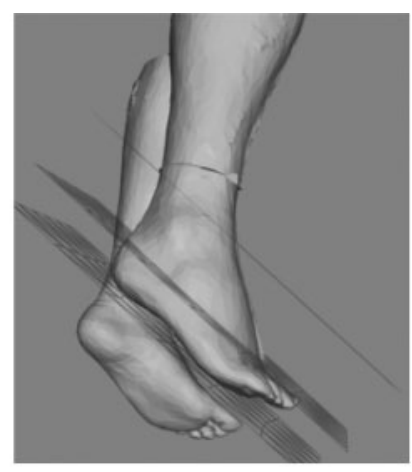

(b) the S or sole offsets

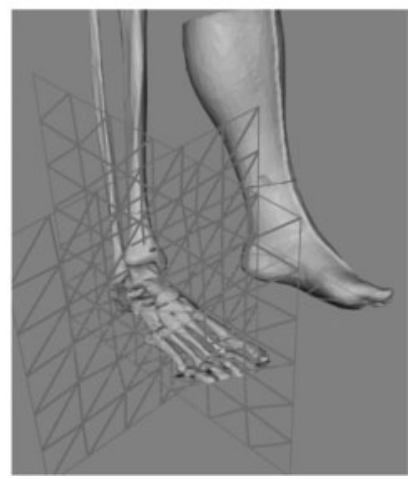

(d) the axis of rotation offsets
Figure 5 The parameterisation planes for the Visible Human male foot component: (a) the B or blade offsets, (b) the $\mathrm{S}$ or sole offsets, (c) the $\mathrm{H}$ or heel offsets, and (d) the axis of rotation offsets.

were subjective, relating to locations that must be decided by the orthotist: the top edge of the orthotic (C3), the bottom edge of skin contact surface on the calf $(\mathrm{C} 2)$ and the lowermost level, where the calf begins to protrude (C1). 
Table 1 Landmark parameterisation of the foot and lower leg

\begin{tabular}{|c|c|c|c|}
\hline Plane & Description & $\begin{array}{l}\text { Offsets }(\mathrm{mm}) \\
\text { for male }\end{array}$ & $\begin{array}{l}\text { Offsets }(\mathrm{mm}) \\
\text { for female }\end{array}$ \\
\hline B0 & Vertical plane tangent to the lateral edge of the foot & 0 & 0 \\
\hline $\mathrm{B} 1$ & The lateral side of the heel & 29.8 & 19.5 \\
\hline B2 & The axis of rotation determined from the skeletal model & 55.5 & 46.2 \\
\hline B3 & The medial side of the heel & 80.7 & 63.9 \\
\hline B4 & Vertical plane tangent to the medial side of the foot & 111.1 & 82.4 \\
\hline S0 & Horizontal plane tangent to the sole of the foot & 0 & 0 \\
\hline S1 & Dorsal surface of toes, distal foot & 28.8 & 46.7 \\
\hline S2 & The axis of rotation for dorsiflexion & 96 & 88.3 \\
\hline S3 & Arbitrary location for height of heel component of the orthotic & 118 & 111 \\
\hline S4 & Derived parameter $(2 \mathrm{~S} 2-\mathrm{S} 3)$, used to establish strap attachment on orthotic & 74 & 65.6 \\
\hline Ho & Vertical plane tangent to posterior heel & 0 & 0 \\
\hline H1 & The axis of rotation for dorsiflexion & 56.3 & 45.8 \\
\hline $\mathrm{H} 2$ & The anterior edge of the heel on the sole & 74 & 67.8 \\
\hline $\mathrm{H} 3$ & The posterior edge of the ball of the foot & 128.5 & 123 \\
\hline $\mathrm{H} 4$ & The meeting of the ball of the foot and the large toe & 216.3 & 198.4 \\
\hline H5 & Derived parameter $(2 \mathrm{H} 1-\mathrm{H} 2)$, used to define vertical strut of the orthotic & 39.1 & 23.7 \\
\hline $\mathrm{C} 0$ & Lower edge of the calf component, ideally coplanar with S2 & 0 & 0 \\
\hline $\mathrm{C} 1$ & Horizontal plane, coincident with first significant change in calf & 83.7 & 85.5 \\
\hline $\mathrm{C} 2$ & Horizontal plane, coincident with bottom edge of skin contact surface of calf & 170 & 161.1 \\
\hline $\mathrm{C} 3$ & Horizontal plane, coincident with intended top of orthotic & 270.9 & 255.7 \\
\hline SH0 & Vertical plane, tangent to the shin & 0 & 0 \\
\hline SH1 & Plane of the axis of rotation, ideally coplanar with $\mathrm{H} 1$ & 21.7 & 29.2 \\
\hline $\mathrm{SH} 2$ & Rear of calf at intersection with $\mathrm{Cl}$ & 91.7 & 98 \\
\hline $\mathrm{SH} 3$ & Rear of calf at intersection with C2 & 112.2 & 111.5 \\
\hline SH4 & Rear of calf at intersection with $\mathrm{C} 3$ & 147.3 & 121.1 \\
\hline SH5 & Derived parameter $(\mathrm{SH} 1 / 2)$, for placement of front edge of calf strut & 10.9 & 14.6 \\
\hline SH6 & Derived parameter, $(\mathrm{SH} 2+\mathrm{SH} 1) / 2$, for placement of back edge of calf strut & 56.7 & 63.6 \\
\hline SH7 & Derived parameter (SH4 + Constant), for placement of rear edge of orthotic & 151.3 & 125.1 \\
\hline L0 & Vertical plane, tangent to lateralmost edge of lower leg & 0 & 0 \\
\hline L1 & Lateral side position of ankle joint, ideally coplanar with B0 & 13.7 & 24.9 \\
\hline $\mathrm{L} 2$ & Derived parameter $(\mathrm{L} 1+\mathrm{B} 2)$ for fitting of ankle hinge & 69.2 & 71.1 \\
\hline L3 & Derived parameter $(\mathrm{L} 1+\mathrm{B} 4)$ for fitting of ankle hinge medial side & 124.8 & 107.3 \\
\hline L4 & Medialmost edge of lower leg & 139 & 115.1 \\
\hline
\end{tabular}

\section{RESULTS}

Table 1 displays the plane designations, descriptions and the offset measurement for each parameter plane of the right foot of the Visible Human male and female. The corresponding design of the heel component, calf component and entire orthotic assembly are displayed in Figure 6, with (a), (b) and (c) representing the fitted orthotic for the male and (d), (e) and (f) representing the fitted orthotic for the female. Qualitatively, the orthotic for the female data set appeared fitted for a more compact foot, with most of the calf mass offset to the lateral side of the joint. By contrast, the male data set resulted in a broader, flatter foot orthotic and a more symmetric lower-leg component.

\section{DISCUSSION}

The parameter-based orthotic design was successfully rescaled to the fitting sizes and axis positions measured from the Visible Human male and female respectively.
While patient comfort cannot be verified, the standardisation of technique suggests the method should be transferable to living patients with CT data of the lower leg and foot. The design used in this sample was extremely simple, and aesthetic and ergonomic improvements may be made with the addition of new parameters. For instance, the foot component may be narrowed at the heel to allow the orthotic to fit within a shoe. Other elements of the design that may be changed include ergonomic factors such as the offset between the orthotic and skin surface or the width of anchor straps.

The actual parameterisation once the 3D model was established was fairly rapid, all offset planes being measured within 15 minutes for each data set after conversion to .3DS format. Another aspect of the parameterisation, that of $3 \mathrm{D}$ reconstruction, likely would have been faster had the imaging data originally been collected for the purpose of fitting. For instance, the Visible Human male images were apparently collected in two different scanning runs, with different positioning of the subject. These slice images had 


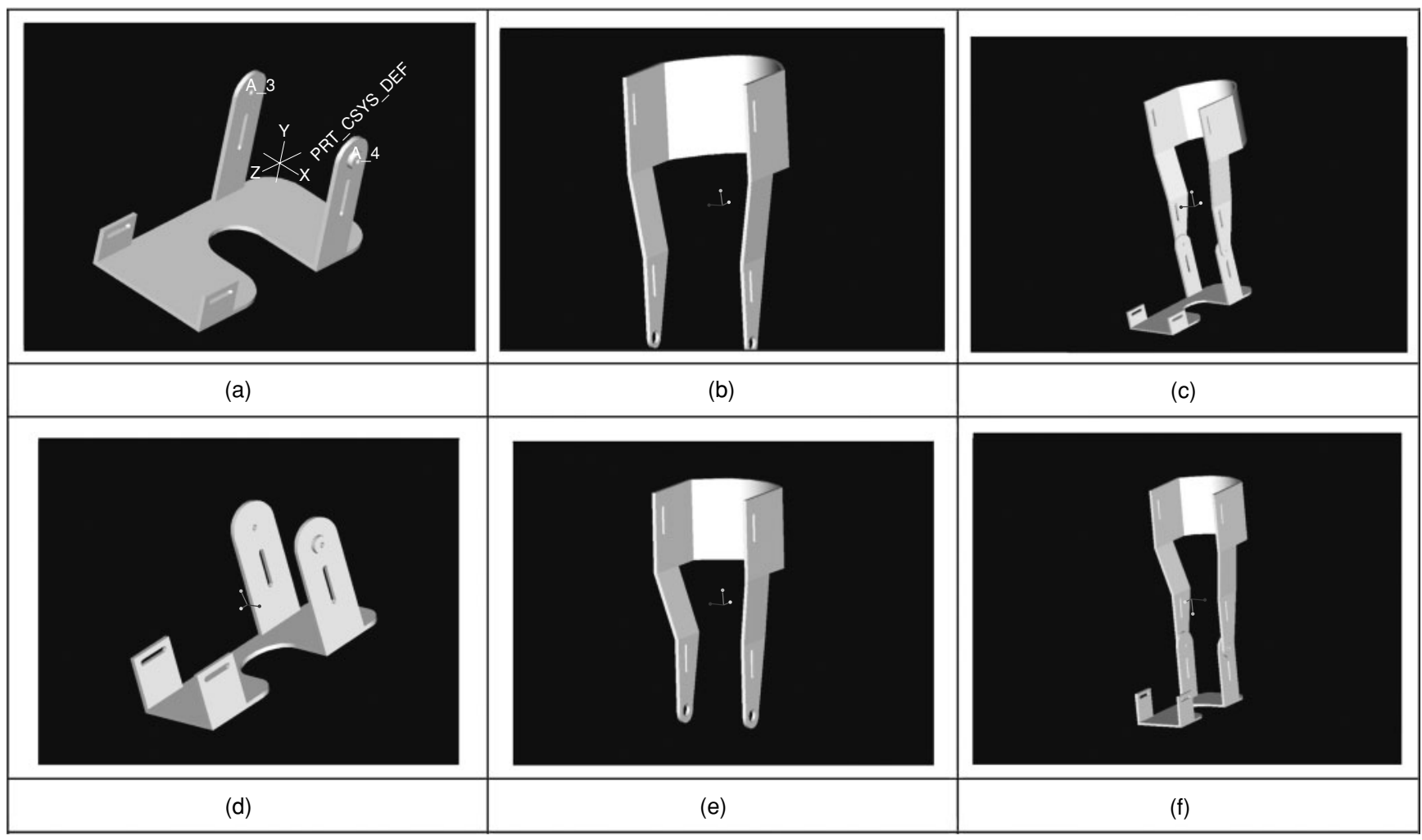

Figure 6 The parameterised orthotic for the Visible Human male (a, b, c) and Visible Human female (d, e, f): the foot component $(\mathrm{a}, \mathrm{d})$, the lower-leg component $(\mathrm{b}, \mathrm{e})$, and the assembly $(\mathrm{c}, \mathrm{f})$.

to be translated to combine the resulting models. In addition, the positioning of the Visible Human male was not conducive to joint parameterisation. In addition to being nearly fully plantarflexed, the foot was also pronated and slightly abducted in its frozen position. As such, certain coordinate planes that should have been coplanar in the lower-leg and foot parameterisations were not. In the female data set, the foot was positioned more cooperatively, and the coordinate systems of the foot and lower leg were only slightly askew. For the sake of orthotic fitting, the patient's foot could be intentionally positioned appropriately for a single CT scanning run.

As another issue of concern, CT scans expose the patient to ionising radiation, prompting ethical concerns about such exposure for the sake of fitting an orthotic. This would suggest that an orthotic should be fit this way only if manual techniques are insufficient. In the case of an ankle orthotic, a CT scan might well be an unacceptable risk, but for a more complicated joint, such as a three-degree-offreedom hip or shoulder, a CT scan might be superior to external manual techniques. Another alternative is MRI, which does not expose the patient to $\mathrm{X}$-rays (Sun and Lal 2002). A drawback of medical imaging in either case, however, is that the patient's limbs tend to deform as the patient lies down to be scanned. An orthotic based on this deformed scan might not be comfortable, so some method would have to be devised to maintain the exterior shape of the limb during scanning. This might include forming a plaster mold over the limb, as is performed in manual fitting, but it would add a further complicating step to the medical imaging fitting approach.

Our selection of treating ankle joint dorsiflexion as a simple hinge is debatable and has been described as an oversimplification in the literature (Leardini 2001). A truer model of the ankle joint complex in terms of dorsiflexion/plantar flexion may be a 2D four-bar linkage model, in which the axis of rotation itself moves during gait, a model with experimental validation (Leardini et al. 1999, Siegler et al. 1998). The change of position of the axis depends on recruitment of individual ligaments to deform and take portions of the load, and as such differs between individuals. For instance, individual differences in foot landing position, from $-12^{\circ}$ (toe in) to $+29^{\circ}$ (toe out) in the general population, cause dramatic differences in this change of axis position (Simpson and Jiang 1999).

\section{CONCLUSION}

Not intended as a direct clinical application, this demonstration illustrated the capacity of $3 \mathrm{D}$ reconstruction methods to parameterise both the exterior and the skeletal structure of CT data sets to refit an orthotic design in a digital format in an automated manner. The simple orthotic design developed for the study showed robust transformation 
between parameterisations between the two CT data sets. The output format of the Pro/ENGINEER orthotic design is compatible with a number of freeform fabrication techniques, or fabrication of part molds. These concepts show potential for expanded use, creating rapidly customisable orthotics for other joints for which medical imaging may be more ethically acceptable, or in creating complex multijoint orthotics requiring more complex fabrication than polymer casting.

\section{ACKNOWLEDGMENTS}

The authors acknowledge the Visible Human Project for the contribution of CT data sets, Michael McDonnell for initiation of the orthotic project, and Dr Sorin Siegler for important consultation.

\section{REFERENCES}

Corazza F, O’Connor JJ, Leardini A, Parenti Castelli V. 2003. Ligament fibre recruitment and forces for the anterior drawer test at the human ankle joint. $\mathcal{F}$ Biomech, 36: 363 72.

Fay BT, Boninger ML. 2002. The science behind mobility devices for individuals with multiple sclerosis. Med Eng Phys, 24: 375-83.

Goldberg B, Hsu JD. 1997. Atlas of Orthoses and Assistive Devices. 3rd ed. St Louis: Mosby.
Leardini A, O’Connor JJ, Catani F, Giannini S. 1999. A geometric model of the human ankle joint. $\mathcal{F}$ Biomech, 32(6): 585-91.

Leardini A. 2001. Geometry and mechanics of the human ankle complex and ankle prosthesis design. Clin Biomech, 16(8): 706-9.

Moseley AM, Crosbie J, Adams R. 2003. High and low-ankle flexibility and motor task performance. Gait Posture, 18: 73-80.

MRIcro. Accessed 2003. URL: http://www.sph.sc.edu/comd/rorden/ mricro.html

Rubin G, Cohen E. 1988. Prostheses and orthoses for the foot and ankle. Clin Podiatr Surg Med, 5(3): 695-719.

Siegler S, Chen J, Schneck CD. 1998. The three-dimensional kinematics and flexibility characteristics of the human ankle and subtalar joints-Part I: Kinematics. $\mathcal{F}$ Biomech Eng, 110(4) 364-73.

Simpson KJ, Jiang P. 1999. Foot landing position during gait influences ground reaction forces. Clin Biomech, 14(6): 396-403.

Sun W, Lal P. 2002. Recent development on computer aided tissue engineering — a review. Comput Methods Programs Biomed, 67: 85-103.

Visible Human Project. 2003. United States National Library of Medicine. Accessed 2003. URL: http://www.nlm.nih.gov/ research/visible/visible_human.html

Wu G, Siegler S, Allard P, Kirtley C, Leardini A, Rosenbaum D, Whittle M, D'Lima DD, Cristofolini L, Witte H, Schmid O, Stokes I. 2002. ISB recommendation on definitions of joint coordinate system of various joints for the reporting of human joint motion-Part I: Ankle, hip, and spine. 7 Biomech, 35: 543-48. 

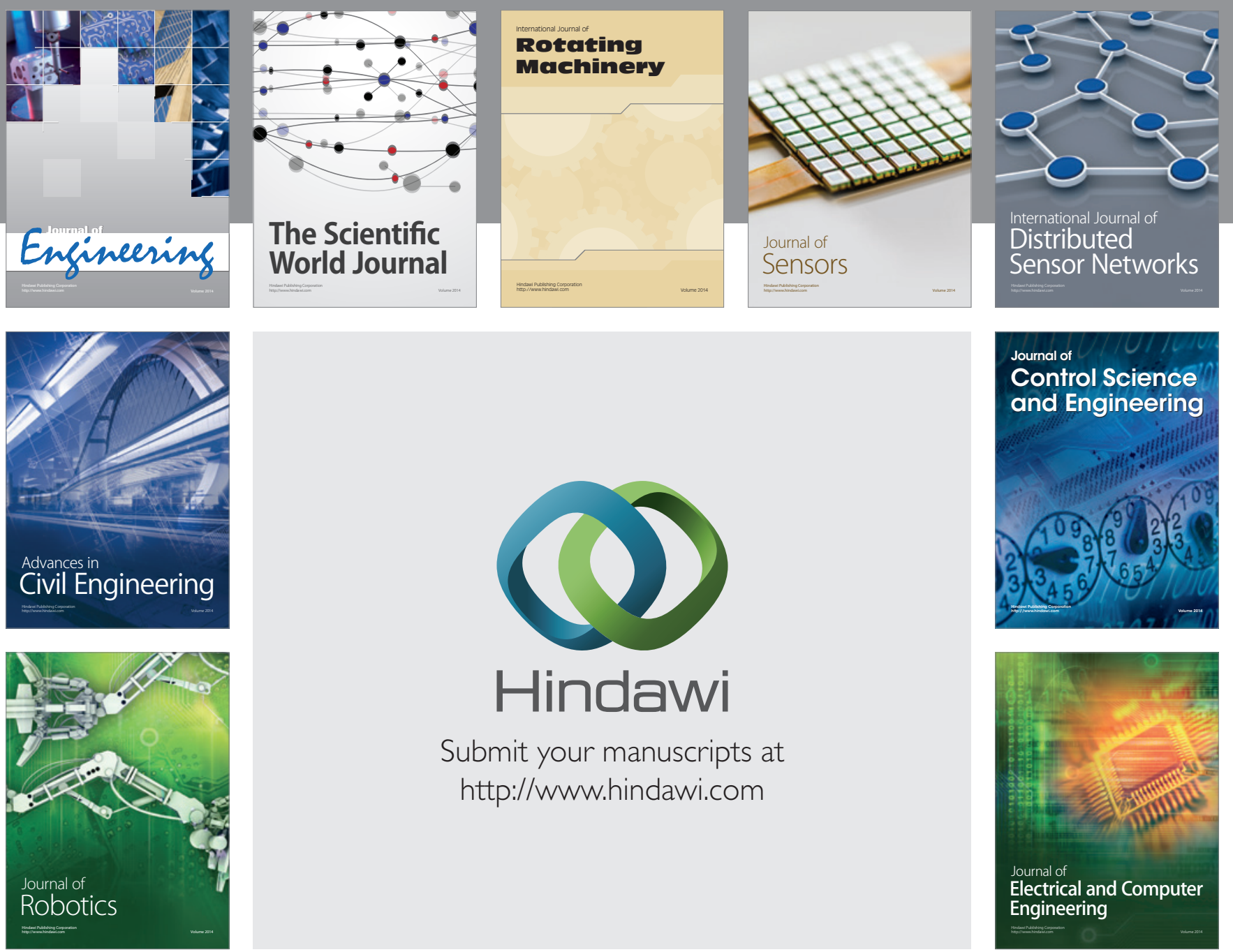

Submit your manuscripts at

http://www.hindawi.com
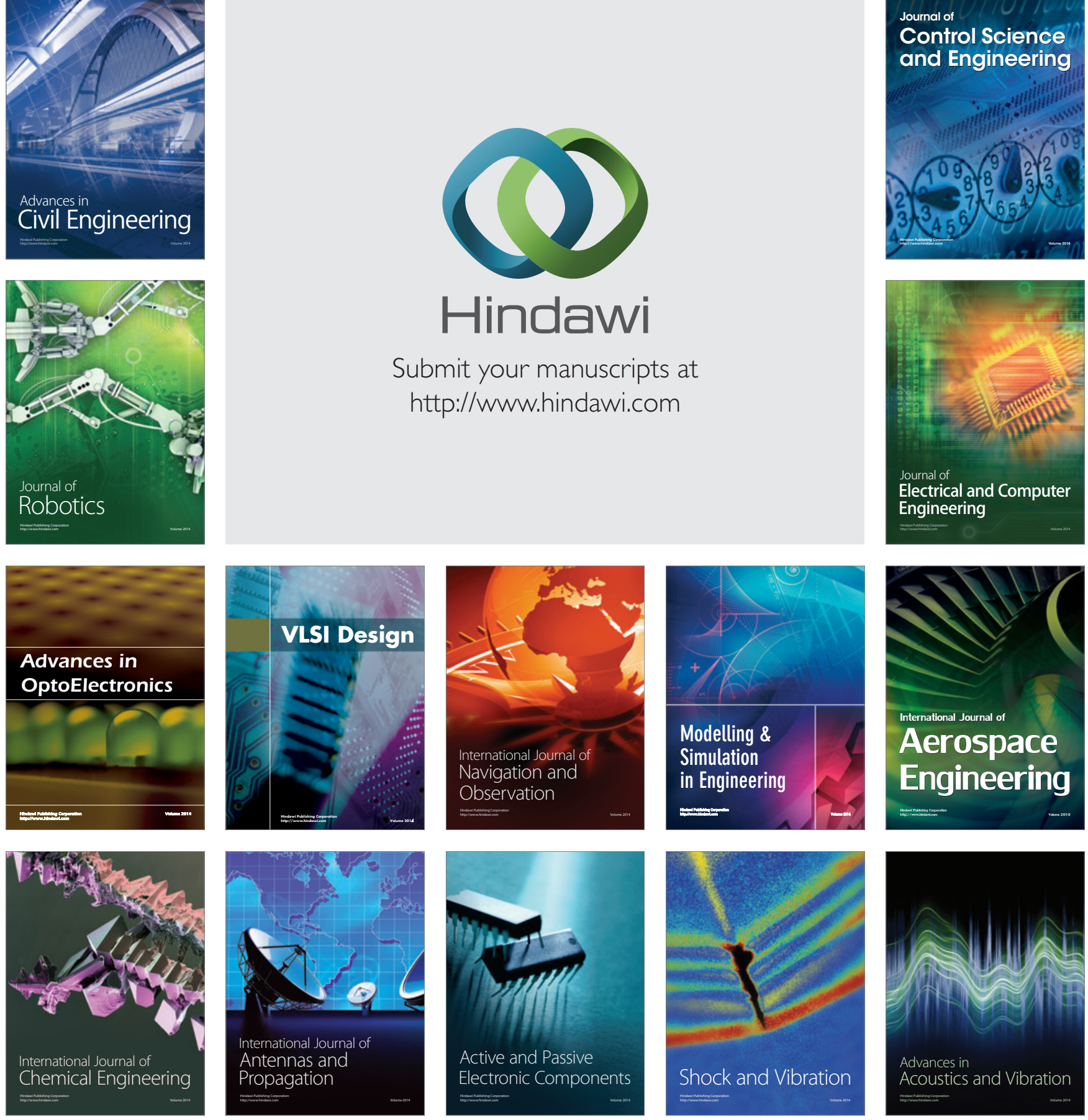